\title{
Recent Insight on the Non-coding RNAs in Mesenchymal Stem Cell-Derived Exosomes: Regulatory and Therapeutic Role in Regenerative Medicine and Tissue Engineering
}

\author{
Tarun Pant ${ }^{1}$, Matea Juric ${ }^{2}$, Zeljko J. Bosnjak ${ }^{1,3}$ and Anuradha Dhanasekaran ${ }^{4 *}$ \\ ${ }^{1}$ Department of Medicine, Medical College of Wisconsin, Milwaukee, WI, United States, ${ }^{2}$ Department of Biophysics, Medical \\ College of Wisconsin, Milwaukee, WI, United States, ${ }^{3}$ Department of Physiology, Medical College of Wisconsin, Milwaukee, \\ WI, United States, ${ }^{4}$ Centre for Biotechnology, Anna University, Chennai, India
}

OPEN ACCESS

Edited by: Sanjiv Dhingra

University of Manitoba, Canada

Reviewed by:

Johnson Rajasingh, University of Tennessee Health Science Center (UTHSC), United States Valentina Casieri, Scuola Sant'Anna di Studi Avanzati, Italy

*Correspondence: Anuradha Dhanasekaran anushivan@gmail.com

Specialty section:

This article was submitted to Cardiovascular Biologics and

Regenerative Medicine,

a section of the journal

Frontiers in Cardiovascular Medicine

Received: 07 July 2021 Accepted: 06 September 2021

Published: 01 October 2021

Citation:

Pant T, Juric M, Bosnjak ZJ and Dhanasekaran A (2021) Recent Insight on the Non-coding RNAs in Mesenchymal Stem Cell-Derived

Exosomes: Regulatory and Therapeutic Role in Regenerative Medicine and Tissue Engineering. Front. Cardiovasc. Med. 8:737512.

doi: 10.3389/fcvm.2021.737512
Advances in the field of regenerative medicine and tissue engineering over the past few decades have paved the path for cell-free therapy. Numerous stem cell types, including mesenchymal stem cells (MSCs), have been reported to impart therapeutic effects via paracrine secretion of exosomes. The underlying factors and the associated mechanisms contributing to these MSC-derived exosomes' protective effects are, however, poorly understood, limiting their application in the clinic. The exosomes exhibit a diversified repertoire of functional non-coding RNAs (ncRNAs) and have the potential to transfer these biologically active transcripts to the recipient cells, where they are found to modulate a diverse array of functions. Altered expression of the ncRNAs in the exosomes has been linked with the regenerative potential and development of various diseases, including cardiac, neurological, skeletal, and cancer. Also, modulating the expression of ncRNAs in these exosomes has been found to improve their therapeutic impact. Moreover, many of these ncRNAs are expressed explicitly in the MSC-derived exosomes, making them ideal candidates for regenerative medicine, including tissue engineering research. In this review, we detail the recent advances in regenerative medicine and summarize the evidence supporting the altered expression of the ncRNA repertoire specific to MSCs under different degenerative diseases. We also discuss the therapeutic role of these ncRNA for the prevention of these various degenerative diseases and their future in translational medicine.

Keywords: MSCs, non-coding RNAs, regenerative medicine, tissue engineering, exosomes

\section{INTRODUCTION}

Over the past several decades, incidences of damage, failure, dysfunction of multiple tissues and organs have reached epidemic proportions (1-3). However, scarcity of donors, limited availability of autologous tissues, risk of chronic tissue rejection, lack of a cost-effective therapeutic strategy, and the overall financial burden of caring for affected individuals present challenges in developing advanced therapeutic options (4-9).

The advent of regenerative medicine has provided immense hope to the primary researcher and clinician to use stem cells with a determined aim of rejuvenating the damaged tissues and 
organs (10-12). Numerous animal models have been used to test stem cell types (e.g., embryonic, induced, and adult) over the past years for autologous and allogeneic therapies (13-16). Although having a relatively limited differentiation potential in comparison to the embryonic stem cells (ESCs) and induced pluripotent stem cells (iPSCs), the combination of factors like autologous cell therapy, reduced possibility of teratoma formation, immunological intolerance, xenogeneic contaminations, lack of ethical concern, and the reduced financial burden have made mesenchymal stem cells (MSCs), an ideal choice for the regenerative cell therapy (17-21). Despite the promising therapeutic potential of MSCs in regenerative medicine, few of the critical questions, such as what are the precise molecular mechanisms responsible for providing the host's immune tolerance against MSCs, is the clinical application of MSCs safe, and how can we increase the scalability and yield per batch, remain unanswered and have imposed significant challenges in their clinical translation, prompting researchers to explore alternative tissue regeneration approaches.

Recent studies propose that MSCs profoundly manifest their action by the paracrine secretion of extracellular vesicles (EVs), particularly exosomes (22-25). The unique potential of these MSCs-derived exosomes (MSCs-Exos) to recapitulate the stem cell properties have paved the path for a "cell-free" therapy in the field of regenerative medicine. Furthermore, in recent years, it has been substantially demonstrated that MSCs-Exos cargo is enriched in distinct ncRNAs, specially-microRNAs (miRNAs), long non-coding RNAs (lncRNAs), circular RNAs (circRNAs), and Piwi-interacting RNA (piRNAs), mediating tissue communication, modifying cellular phenotype, regulating wide range of biological, pathophysiological processes making them a relevant drug delivery and therapeutic option in the field of regenerative medicine (26-30).

In the current review article, we aim to summarize the emerging role of MSCs-Exos-derived ncRNAs (MSCs-Exos ncRNAs) in regenerative medicine and diseases. We further emphasize the regulatory mechanisms, therapeutic approaches, ongoing clinical applications, and the challenges associated with MSCs-Exos ncRNAs in the field of regenerative medicine and tissue engineering.

\section{MSC-EXo'S BIOGENESIS}

MSCs are stromal, nonhematopoietic cells generally obtained from different sources including bone marrow, adipose tissue, umbilical cord, placenta, amniotic fluid, and dental pulp (31-37). The characterization of the MSCs depends upon the expression of specific cell surface markers (CD29, CD37, CD73, CD90, CD102, CD105, and CD166) and their potential to differentiate into multiple cell types such as osteocytes, chondrocytes, and adipocytes under appropriate, stimulating culture conditions (38-43). Recent clinical and preclinical trials have demonstrated the potential of MSCs to be an excellent autologous cell source treating numerous diseases including diabetes, myocardial infarction, osteoarthritis, Alzheimer's, both in animal models and patients (44-49). Although MSCs-based therapy has proven beneficial in treating a wide range of diseases, we still have incomplete knowledge regarding their mechanism of action. Emerging studies in recent years have found that MSCs mediate their action via the paracrine secretion of exosomes (Figure 1), regulating numerous biological processes (50).

Exosomes are membrane-enclosed spherical or cup shaped endocytic vesicles with a size ranging from 40 to $150 \mathrm{~nm}$ formed intracellularly in the cellular multivesicular bodies and released from numerous cell types (51-53).

Exosome biogenesis occurs via three pathways: ESCRTdependent, ESCRT independent, or direct budding of the plasma membrane. In the first two pathways, an early endosome is formed by the inward budding of the endosomal membrane to generate intraluminal vesicles (ILVs). These ILVs accumulate to form multivesicular bodies (MVBs), which fuse with the plasma membrane (facilitated by Rab GTPases). The ILVs are then released from cells as exosomes, where they are taken up by recipient cells via endocytosis, direct binding, or ligandreceptor binding. The Endosomal Sorting Complexes Required for Transport (ESCRT) includes four distinct proteins: ESCRT 0, I, II, and III. Briefly, ESCRT 0 relegates ubiquitinated proteins within the endosomal membrane and recruits ESCRT I and II. ESCRT I and II bind near ubiquitinated proteins on the outer surface of the endosomal membrane, "tagging" them for recruitment within newly forming intraluminal vesicles in the MVB. ESCRT III then sequesters MVB proteins, finalizing the process of exosome formation. Exosome biogenesis via the ESCRT-independent pathway was discovered recently after knock-out studies involving the ESCRT complex proteins showed that the cells could continue the process of exosome formation and release. This pathway involves lipids such as sphingolipids and ceramides and proteins such as heat shock proteins and tetraspanins (54-56). Why these distinct pathways exist, and which pathway is used in cells is still poorly understood. Investigators have used different approaches to increase the angiogenic potential of exosomes released by the stem cells (57). The release of exosome can be considerably increased in vitro using stress conditions that mimic organ injuries, such as hypoxia, irradiation, or drug treatments.

The MSC-Exos have been tested widely on human patients of ischemic heart injury, cerebrovascular disease, and liver fibrosis as an alternative to MSCs themselves (58-61). Intriguingly, numerous advantages over MSCs such as increased viability, higher uptake, lower immune response, reduced risk of embolism, and potential to cross the blood-brain barrier have made MSC-Exos a promising candidate emerging as an effective "cell-free" therapeutic approach in the field of regenerative medicine $(22,62-64)$.

In recent years, considerable amounts of research depict MSCs-Exos ability to facilitate the exchange of genetic and epigenetic information intercellularly leading to the modulation of neighboring and distant cells' gene expression in both healthy and disease states (65). Preclinical data further demonstrate that the acquired repertoire of biological cargo, primarily ncRNAs of MSC-Exos, has been found to effectively disseminate biological information between cells and regulate the inflammatory apoptotic pathways in various and disease states $(64,66)$. 

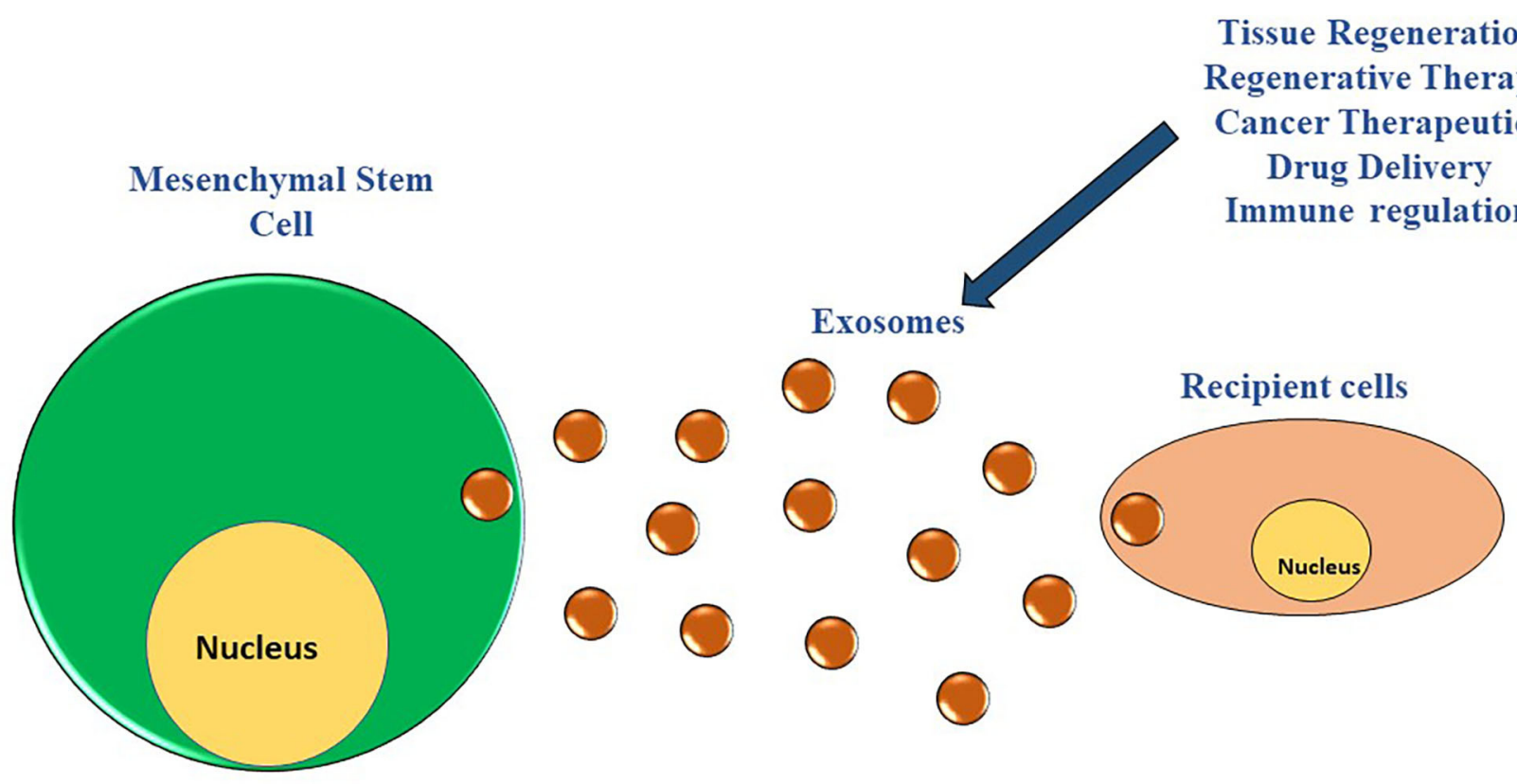

FIGURE 1 | MSCs derived exosomes and their interaction with the neighboring cells. The MSCs have the potential to modulate numerous biological processes of numerous cell types in their vicinity via paracrine secretion of exosomes.

In the next section, we represent the overview of ncRNAs from the MSC-Exos in context of regenerative medicine. We also discuss their role as diagnostic and therapeutic targets for regenerative medicine and tissue engineering.

\section{OVERVIEW OF MSC-EXo-ncRNA}

In the past two decades, advancements in high throughput sequencing technologies and computational approaches have successfully captured and annotated the transcriptome revealing the non-coding transcripts and their functionality in the human genome $(67,68)$. The extensive information from these indepth analyses demonstrates the genome complexity, with $<2 \%$ of the genome encoding than a large percentage (98\%) being transcribed into heterogeneous ncRNAs transcripts (69).

The ncRNAs comprises of a diversified repertoire of endogenous RNA transcripts including short and long $(<200$ nucleotides) ncRNAs with no protein-coding potential (70, 71). Initially, the ncRNAs were considered as transcriptional junk having no biological function; however, in recent years, numerous studies have depicted the regulatory function of ncRNAs modulating the expression of genes involved in critical biological processes (72). Moreover, the dysregulated expression signatures of ncRNAs are found to be contributing toward the pathogenesis of various diseases by diverse mechanisms (Figure 2) (73-77).

The MSCs are active sources of exosomes with enriched cargo, including lipids, DNA, RNAs (mRNAs, miRNAs, lncRNAs, circRNAs, and piRNAs) proteins. MSC-Exos are also being explored as viable biomarker in diseases. Their cargo of noncoding RNA (ncRNA), including lncRNA and miRNA, is being studied for their role as potential therapeutic strategies in regenerative medicine (Table $\mathbf{1}$ ).

\section{ROLE OF MSC-EXO-ncRNA IN REGENERATIVE MEDICINE}

\section{Bone Regeneration}

The regeneration of bone is a dynamic and complicated process that requires the coordination of numerous cell types and biological events such as ossification, osteoinduction, and osteogenesis. In addition to the above-mentioned biological events, series of signaling pathways associated with angiogenesis, proliferation, migration, remodeling, inflammation, and necrosis further play a critical role at the site of bone injury. Bone's inability to regenerate successfully results from the failure in the orchestration of the above-mentioned biological processes and signaling pathways. Bone regeneration failure is one of the significant health problems affecting millions of people worldwide, requiring immediate approaches to improve bone healing. In recent years, studies in regenerative medicine have shown the MSC's potential in promoting bone regeneration through paracrine mechanisms, mainly mediated by exosomes (98-101). The mechanisms governing the beneficial role of MSC-Exos in context to bone regeneration remain, however, elusive.

Current studies have shown that the exosomes from a different source of MSCs mediate ncRNA transport to the injured site to regulate successive biological processes enhancing bone 


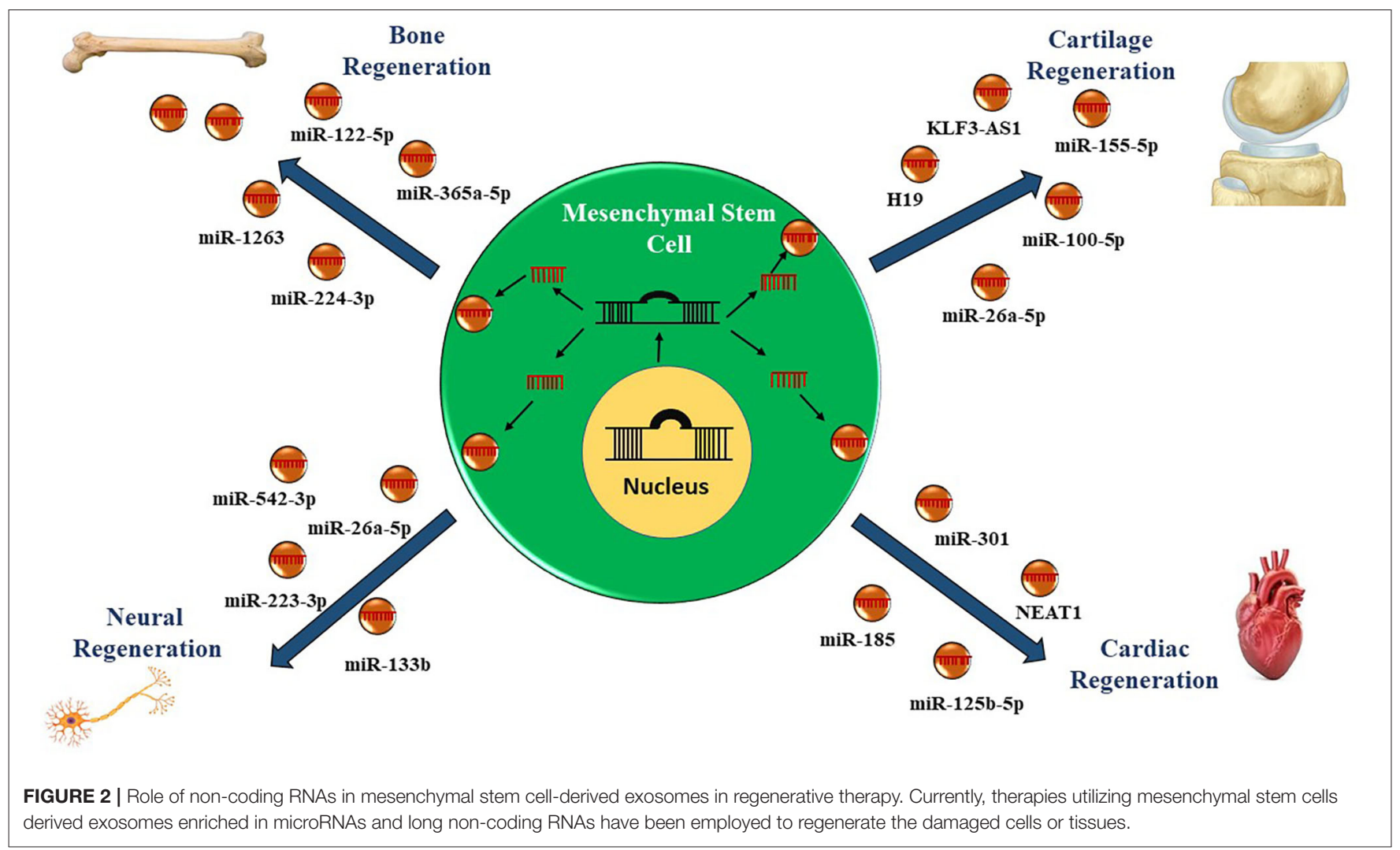

reconstruction. For example, Liao et al. (78) investigated the role of bone marrow MSC (BMSCs)-derived exosomes expressing miR-122-5p on the femoral head's osteonecrosis (ONFH). The authors demonstrated that the expression profile of miR-122-5p was decreased in $\mathrm{ONFH}$, while exosomes carrying miR-122-5p overexpression effectively attenuated $\mathrm{ONFH}$ by downregulating SPRY2 via the RTK/Ras/mitogen-activated protein kinase (MAPK) signaling pathway (78). Another study suggested that decreased expression of miR-224-3p levels in BMSCs-Exos can attenuate traumatic ONFH by facilitating angiogenesis by upregulating focal adhesion kinase family interacting protein (FIP200) in endothelial cells (79). Similarly, exosomal miR-365a$5 \mathrm{p}$ produced by human umbilical cord-derived MSCs (humMSCs) was found to alleviate glucocorticoid (GC)-induced osteonecrosis of the femoral head (GIONFH) by promoting osteogenesis via activation of the Hippo signaling pathway in rats (80). Moreover, in a hind limb unloading (HLU)-disuse osteoporosis (DOP) rat models, hum-MSC-Exos miR-1263 effectively ameliorated the apoptosis of BMSCs via modulating the Mob1/Hippo signaling pathway in the prevention of rat $\operatorname{DOP}(81)$.

Interestingly, research efforts have shown that preconditioning can influence the regenerative efficacy of MSC-derived Exos. Using an in vivo model of bone fracture, the authors illustrate that hypoxia preconditioning of MSCs enhances exosomal miR-126 by activation of hypoxia-inducible factor $1 \alpha(\mathrm{HIF}-1 \alpha)$ and the SPRED1/Ras/Erk signaling pathway. Additionally, knockdown of HIF-1 $\alpha$ resulted in decreased expression of MSCs-Exos miR-126, abolishing its protective effect in bone healing (84).

The effects of age on the MSCs-Exos on osteogenic differentiation, osteogenesis, and fracture healing have also been investigated. Bone regeneration is often associated with age, characterized by decreased bone formation due to reduced osteogenic differentiation. Notably, a negative correlation was reported in the expression of miR-128-3p derived from MSCsExos and bone regeneration. This in vitro experiment further reveals that exosomal miR-128-3p can suppress bone healing by inhibiting the expression of Smad5. Furthermore, reverting the effect of miR-128-3p using antagomir was found to enhance the bone recovery process via the increase in Smad5 (83). Additionally, BMSCs exosomal miR-186 promoted osteogenesis via MOB Kinase Activator 1A (Mob1)/Hippo signaling pathway in the ovariectomized (OVX) rats model of postmenopausal osteoporosis (PMO) (82).

In conclusion, the above research results substantiate that the beneficial effect of MSC-Exos is mediated by ncRNAs, making them a potential therapeutic candidate in bone regeneration.

\section{Cartilage Regeneration}

In the recent years, the therapeutic roles of exosomes secreted from MSCs have also been assessed regarding cartilage regeneration. Interestingly, the exosomal vesicles derived from MSCs can regulate proliferation, differentiation, and matrix synthesis, indicating this strategy's significance in cartilage regeneration $(102,103)$. Exosomes' therapeutic mechanisms 
TABLE 1 | Mesenchymal stem cell-derived exosomes non-coding RNAs in bone regeneration application.

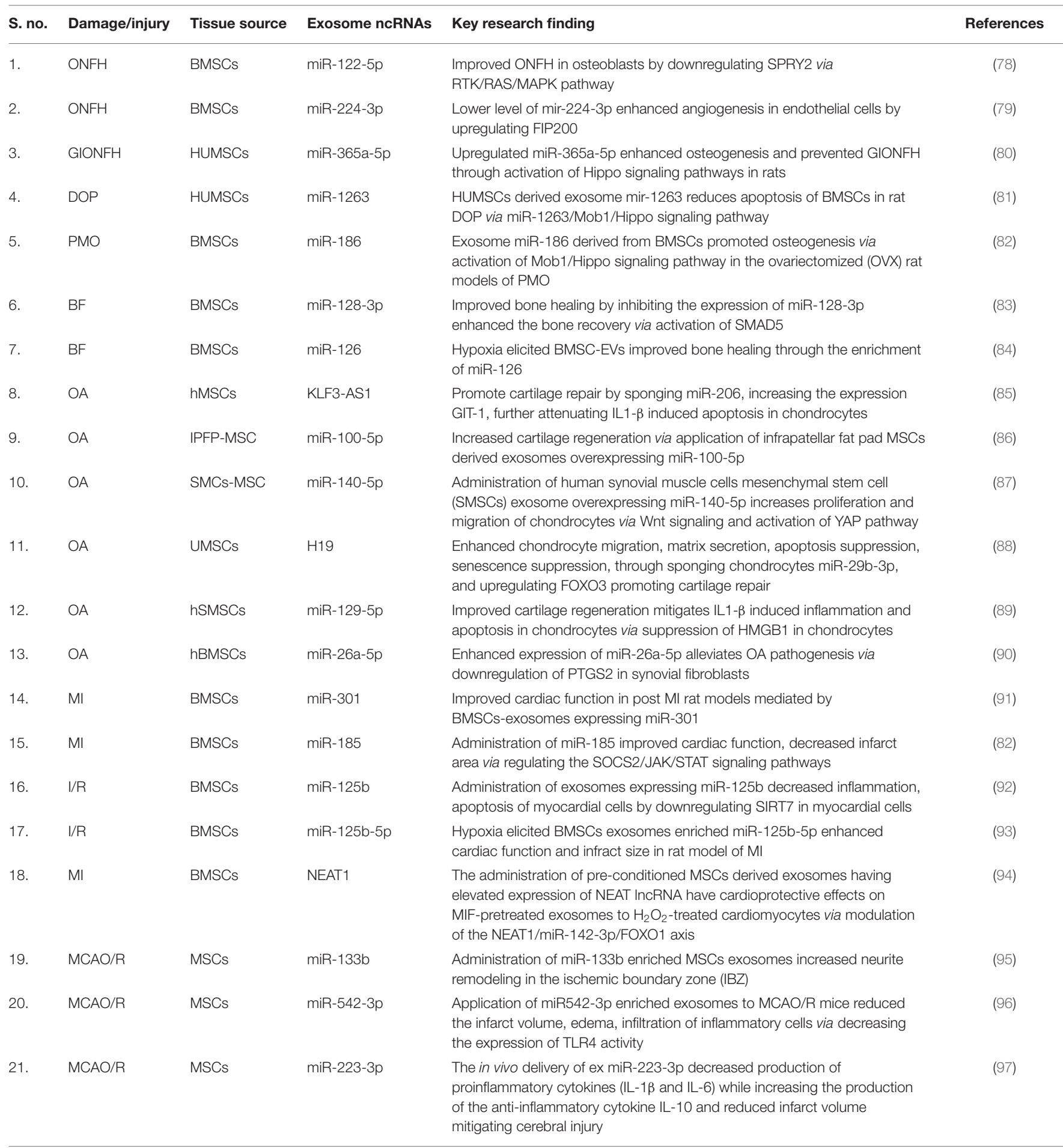

ONFH, Femoral head's osteonecrosis; GIONFH, Glucocorticoid (GC)-induced osteonecrosis of the femoral head; DOP, Disuse osteoporosis; PMO, Post-menopausal osteoporosis; BF, Bone fracture; MAPK, Mitogen Activated Protein Kinase; FIP, Family interacting protein; Mob1, Mob Kinase Activator 1; SMAD5, Mothers against decapentaplegic homolog 5; KLF3-AS1, KLF3 antisense RNA-1; IPFP-MSC, Infrapatellar fat pad-derived mesenchymal stem cell; GIT1, G-protein-coupled receptor kinase interacting protein-1; YAP, Yes-associated protein; FOXO3, Forkhead box O3; HMGB1, High Mobility Group Box 1; PTGS2, Prostaglandin-endoperoxide synthase 2; MI, Myocardial Infraction; IR, Ischemia Reperfusion; SOCS2, Suppressor Of Cytokine Signaling 2; JAK, Janus Kinase 1; STAT, signal transducer and activator of transcription; SIRT7, NAD-dependent deacetylase Sirtuin 7; MCAO/R, Middle cerebral artery occlusion reperfusion. 
through ncRNAs in context to cartilage regeneration have, however, only more recently begun to be explored. Recent data suggest that cytokines' inflammatory effect can be regulated by the EVs derived from MSCs containing lncRNA. For instance, the protective effect of exosome lncRNA-KLF3-AS1 derived from hMSCs was investigated in the collagenaseinduced rat model of osteoarthritis (OA) and IL- $1 \beta$-induced OA chondrocytes. The IncRNA-KLF3-AS1 could exert a therapeutic effect by suppressing IL- $1 \beta$-induced apoptosis in chondrocytes. In addition, in vivo investigation further indicated that exosomal KLF3-AS1can promote cartilage repair and chondrocyte proliferation in a rat model of OA (104). These outcomes were mediated by sponging the miR-206 and increasing the expression of G-protein-coupled receptor kinase interacting protein-1 (GIT1) in the chondrocytes (85).

Interestingly, the application of infrapatellar fat pad (IPFP) MSCs-derived exosomes (MSC ${ }^{\mathrm{IPFP}}$-Exos) overexpressing miR$100-5 p$ were reported to diminish cartilage deterioration via inhibition of the mTOR-autophagy pathway (86). In a rat model of OA, administration of human synovial muscle cells mesenchymal stem cells (SMSCs) exosomes overexpressing miR$140-5 \mathrm{p}$ was found to increase the proliferation and migration of chondrocytes via Wnt signaling and activation of Yesassociated protein (YAP) in a rat model of OA (87). Similar results were reported when the SMSCs exosomes expressing miR155-5p showed an anti-apoptotic response in the osteoarthritic chondrocytes and further promoted proliferation and migration, enhanced ECM secretion, and effectively prevented $\mathrm{OA}$ in a mouse model (105).

In a separate study, umbilical cord mesenchymal stem cells (U-MSCs) exosomes attenuated osteochondral damage via upregulation of lncRNA H19 (88). Further investigation of the role of U-MSCs exosomes demonstrated their ability to transfer the lncRNA H19 to chondrocytes. The exosomederived lncRNA H19 was notably successful in promoting chondrocyte migration, matrix secretion, apoptosis suppression, as well as senescence suppression, both in vitro and in vivo by acting as a competing endogenous sponge against miR-29b3 p upregulating FoxO3 (106). To explore the anti-inflammatory effect of exosomes in $\mathrm{OA}$, it was revealed that human synovial mesenchymal stem cells (HS-MSCs) exosomes-derived miR-129$5 p$ could mitigate interleukin-1 $\beta$ (IL-1 $\beta$ )-induced inflammation and apoptosis in chondrocytes via suppressing the expression of high mobility group box protein-1 (HMGB1) (89). In another study on a rat model hBMSCs-derived exosome overexpressing miR-26a-5p improved OA pathogenesis via downregulating prostaglandin-endoperoxide synthase 2 (PTGS2) expression in synovial fibroblast (90). In another work, the authors recently demonstrated the anti-inflammatory and chondroprotective effect of hBMSCs-derived exosome miR-26a-5p attenuating OA via regulation of syndecan-1 (SDC1) (107).

In short, these findings suggest the therapeutic potential of MSCs-Exos ncRNAs in cartilage regeneration.

\section{Cardiac Regeneration}

Mesenchymal stem cells (MSCs) have been studied in great depth regarding their potential use in regenerative medicine. The most notable application is the regeneration of cardiac tissue after myocardial infarct (MI) (108). Cardiomyocyte regenerative capacity is markedly limited as these cells are not thought to enter the cell cycle to undergo division. As a result, injured tissue generally becomes necrotic, and a non-contractile scar tissue forms, consequently limiting the contractile ability to neighbor cells. Due to their limited capacity to regenerate, cardiomyocytes have been the target of recent advents in regenerative medicine as MSCs have been shown to direct cardiac tissue reconstruction following MI $(109,110)$. Previously, it was suggested that MSCs differentiate into cardiomyocytes and that this process was responsible for repairing cardiac tissue (111, 112). This hypothesis turned out not to be feasible. However, there are some cardiac benefits from treatment with stem cells, including decreased cardiomyocyte apoptosis, reduced fibrosis, enhanced neovascularization and improved left ventricular ejection fraction; due to the exosomes released from the stem cells in situ. Because of this effect exosome therapy recapitulates the benefits of stem cell therapy (57), and studies have shown that the activation of cardioprotective pathways obtained by stem cell therapy can be recapitulated by the administration of exosomes obtained from the stem cells (113). Moreover, some studies have demonstrated that exosomes released from cardiac progenitor cells can improve cardiac function in the damaged heart $(114,115)$.

Recent evidence points to MSC-derived exosomal cargo, including lncRNA and miRNA, which impart these regenerative effects via paracrine action on nearby cardiomyocytes (27, $116,117)$. Following this observation, exosomes have become desirable therapeutic avenues in regenerative medicine due to their low immunogenicity, low toxicity, stability, and cargo durability.

$\mathrm{Li}$ et al. (91) discovered that bone marrow-derived MSC (BM-MSC) exosomes expressing miR-301 protected rat cardiomyocytes from autophagy following $\mathrm{MI}$ and improved cardiac function in post-MI rat models. Compared to sham rats, exosome miR-301-treated rats showed higher left ventricular ejection fractions (LVEF) and left ventricular fractional shortening (LVFS) as well as decreased left ventricular end diastolic diameter (LVEDD) and left ventricular end systolic diameter (LVESD) following myocardial infarct. These variables are commonly assessed to measure cardiac function. The LC3II/LC3-I ratio decreased in exosome miR-301 rat models post myocardial infarct and p62 expression increased, indicating a lower level of autophagy compared to sham models (91).

In another study, administration of BM-MSCs exosomes harboring miR-185 inhibited SOCS2, a member of the SOCS family, which function as repressors of JAK/STAT signaling. SOCS2 is a feedback suppressor of the growth hormone/insulinlike growth factor axis. In mouse models, increased miR-185 decreased the expression of SOCS2 and exosome derived miR185 delivered to mice post-MI improved cardiac function, decreased collagen I and III, and decreased fibronectin while also decreasing infarct area. Overexpression of SOCS2 reversed the regenerative effects of miR-185. miR-185 decelerated the rate of apoptosis in cardiac tissue post-MI with an observed decrease in BAX expression and increase in Bcl-2 expression (118). 
Similar to the effects seen in miR-301 expressing exosomes, Chen et al. observed that exosomes harboring miR-125b increased viability and decreased inflammation and apoptosis of ischemia/reperfusion injury (I/R) rat model myocardial cells by downregulating SIRT7 (92). SIRT7 is in the sirtuin family and functions as a deacetylase to preserve the repressive action of heterochromatin (119). SIRT7 has also been implicated as an apoptosis regulator by hyperacetylating p53 in vivo (120).

At the forefront of exosome therapeutic efficacy lie barriers to mimic the exact clinical condition and specific delivery to the affected tissue, limiting the therapeutic efficacy and clinical translation. The pre-conditioning in environments and further conjugating the exosomes to targeted proteins provides another route of establishing desirable qualities exosomes which can be delivered specifically $(121,122)$.

Recently, Zhu et al. demonstrated a novel mechanism of conjugating CSTSMLKAC peptide [derived from ischemic myocardium-targeted (IMT) peptide], interacting with cardiac troponin 1- to hypoxia-conditioned mouse BM-MSCs (Hypoexo). The IMT-conjugated exosomes were specifically targeted to myocardial tissue and retained in the ischemic left ventricle vs non-ischemic right ventricle as observed $24 \mathrm{~h}$ post-MI. The BMMSCs (Hypo-exo) were enriched in anti-apoptotic miR-125b$5 \mathrm{p}$. Intramyocardial delivery of Hypo-exo reduced infarct size, improved cardiac function, and significantly decreased apoptosis in mice with permanent-condition MI. Exosome miR-125b-5p treated rats showed increased cardiac function and decreased infarct size compared to control-exosome-treated rats. The pre-conditioning in environments mimicking cardiac ischemia as opposed to exogenously incorporating non-coding RNAs provides another route of establishing desirable qualities in MSCderived exosomes (93).

Beyond miRNA cargo, lncRNAs such as NEAT1 have been implicated in cardio protection as upregulation of NEAT1 has shown cytoprotective effects in doxorubicin-induced cardiac injury (94). Interestingly one of the studies reported that NEAT1 sponges miR-142-3p, known to regulate FOXO1 expression in an inhibitory manner. FOXO1 belongs to the FOXO family of transcription factors that function to ameliorate oxidative stress and inflammation and increase expression of antioxidant enzymes such as superoxide dismutase (SOD) and catalase in many cell types including cardiac tissue (123).

In another study preconditioning of MSC-derived exosomes with macrophage migration inhibitory factor (MIF), known to be upregulated in cardiac tissue following $\mathrm{MI}$, led to increased levels of NEAT1 lncRNA in pre-conditioned exosomes. Cardioprotective effects were seen following administration of MIF-pretreated exosomes to $\mathrm{H}_{2} \mathrm{O}_{2}$-treated cardiomyocytes via modulation of the IncRNA NEAT1/miR-142-3p/FOXO1 axis as seen with decreased levels of caspases $3 / 7$ and 8 , decreased reactive oxygen species (ROS) production and lipid peroxidation, and increased SOD expression (124).

Research continues to delve into MSC-derived exosomes' intricacies as both in vitro and in vivo studies have shown the cytoprotective affects and regenerative capabilities associated with exosome-derived ncRNA cargo. There is much to be learned regarding interactions of exosome-derived ncRNA and host targets. Importantly, discerning the risks associated with delivering anti-apoptotic ncRNA and the potential risk of tumorigenesis of host tissue remains elusive. Targeted exosome delivery to affected organs continues to be explored as this remains a barrier to transitioning exosome-derived therapeutics to clinical settings. It would be interesting to conduct more randomized controlled trials on larger animals other than rats/mice. Taken together all the above findings indicates the role of MSCs-Exos ncRNAs in cardiac regeneration.

\section{NEURODEGENERATIVE DISEASES}

Neurodegeneration is a persistent barrier to the effective recovery of patients following a cerebrovascular accident (CVA) with ischemic stroke (IS). According to the American Heart Association, IS has become the fifth leading cause of death in the United States (125). A blockage in the cerebral arteries characterizes ischemic stroke, predominantly occurring in the middle cerebral artery (MCA), which subsequently prevents blood flow to the brain. Thrombolytic therapies are the firstline interventions administered following an ischemic stroke, but their effectiveness decreases as the time from stroke to hospital admission increases (126). Lack of oxygen following blockage of the cerebral artery causes downstream cerebral tissue hypoxia and cell death, leading to irreversible damage to brain tissue and accompanying neurocognitive disabilities (127). Researchers have been working to regenerate injured brain tissue following IS using mesenchymal stem cell-derived exosomes as increasing evidence has pointed to the therapeutic role of miRNAs in attenuating cell damage and inflammation following brain injury (128-131).

A primary goal in treating ischemic stroke is decreasing compensatory injury to nerve cells deprived of oxygen downstream of the vascular blockage. Rapid reperfusion is performed to reinstate oxygen to the affected tissue to rescue cells from hypoxia-induced damage, though only a limited time window is allotted for effective reperfusion. Various miRNAs are involved in the mitigation of ischemic stroke, whereby their exogenous introduction to tissues via exosomes has been shown to remediate damages associated with CVAs. Xin et al. (95) observed that $\mathrm{MCAO} / \mathrm{R}$ rats exhibited improved functional outcomes and increased neurite remodeling in the ischemic boundary zone (IBZ) following administration of miR-133b-enriched MSC exosomes.

Inflammatory processes in hypoxic tissue pose another hurdle to effective recovery of patients following IS as persistent activation of inflammatory mediators results in tissue edema and prolonged recruitment of leukocytes to the affected area (132). Cytokines released from injured neural tissue activate inflammatory pathways, of importance being the NF$\mathrm{kB}$ pathway, known for producing proinflammatory cytokines such as IL-1, IL-2, IL-12, and TNF- $\alpha$, among others. Toll-like receptor 4 (TLR4) is a PRR located on the cell surface of microglia and other macrophages and is involved in intracellular signaling via the NF-kB pathway (133). Cai et al. (96) showed that decreasing the expression of TLR4 via delivery of miR-542-3p 
enriched MSC-exosomes to $\mathrm{MCAO} / \mathrm{R}$ mice reduced infarct volume, edema, and infiltration of inflammatory cells.

Augmenting the inflammatory response through controlling microglial polarization is an attractive route for mitigating cerebral injury following IS. Microglia are macrophages that are key players during the inflammatory process during and after IS and are responsible for both innate and adaptive immunity in the brain. Their polarization into either the M1 or M2 phenotype is characterized by pro-inflammatory or anti-inflammatory cytokine production, respectively (134). Zhao et al. were able to induce M1 to M2 microglial polarization in BV2 mouse microglial cells via the introduction of miR-2233p-enriched MSC-exosomes (ex miR-223-3p). miR-223-3p was found to decrease cysteinyl leukotriene receptor 2 (CysLT2R), which may play a role in the induction of the M1 macrophage phenotype. Treatment with ex miR-223-3p on BV2 cells induced their conversion from the M1 to the M2 phenotype while in vivo delivery of ex miR-223-3p decreased production of pro-inflammatory cytokines (IL-1 $\beta$ and IL-6) while increasing the production of the anti-inflammatory cytokine IL-10 and reduced infarct volume in conjunction with improving the functional recovery of rats, including activity, learning, and memory (97).

Neurodegeneration remains an imminent barrier to the recovery of patients following IS. Exosome-derived non-coding RNAs serve many biological processes and are critical mediators in the regeneration and protection of nerve tissue following IS-induced brain injury.

\section{CANCER THERAPEUTICS}

MSC-derived exosomes are currently being explored as viable biomarkers in disease and their cargo of non-coding RNA (ncRNA), including lncRNA and miRNA, are being studied for their role as potent therapeutic strategies in regenerative medicine. This RNA cargo is also being explored in the context of cancer therapeutics while the exosomal skeleton is being looked at as a possible vehicle for delivery of chemotherapeutic agents. The role of MSC-derived exosomes in cancer is quite a contested subject, as numerous reports have provided evidence that various MSC-exosomal lncRNA and miRNA can either inhibit cancer cell proliferation/induce apoptosis or induce metastasis/cancer progression (135-140).

In the recent years, KRAS targeting has emerged to be an efficient therapeutic efficacy in context to lung and pancreatic cancer as KRAS mutations are found in 30\% of lung and $90 \%$ of pancreatic carcinomas. Yet, KRAS has been denoted an "undruggable" target (in part due to its high affinity for GTP/GDP, smooth surface with limited binding pockets, lack of allosteric binding sites, and extensive post-transcriptional modifications. H3K27me3 methylation is associated with the downregulation of nearby genes, of importance being KRas (involved in the Ras/MAPK pathway) (141-143). Xu et al. (135) demonstrated that miR-124 from bone marrowderived mesenchymal stem cells (BM-MSCs) directly inhibited expression of EZH2, which suppressed proliferation, migration, and epithelial mesenchymal transmission (EMT) while inducing apoptosis in pancreatic cancer cells (Aspc-1 and PANC-1).

$\mathrm{Xu}$ and colleagues found that MSC-derived exosomal miR$133 \mathrm{~b}$ inhibited proliferation, migration, and invasion of U87 glioma cells by decreasing EZH2 expression in vitro and inhibited tumor growth in vivo via modulating the Wnt/ $\beta$ catenin signaling pathway by inhibiting the expression of EZH2 (136). In another study, miR-133b was shown to suppress glioblastoma invasion and cell migration via downregulation of metalloproteinase 14 (MMP14) in U87 and U251 glioma cells, while miR-133b was also shown to impede proliferation and invasion of glioma due to Sirt1 downregulation $(144,145)$.

Pakravan et al. found that MSC-derived miR-100 suppressed angiogenesis in breast cancer cells by altering the mTOR/HIF$1 \alpha /$ VEGF signaling axis. mTOR is a protein kinase involved in the $\mathrm{PI} 3 \mathrm{~K} / \mathrm{AKT}$ pathway and is known to drive HIF-1 $\alpha$ accumulation, which acts as a transcription factor by binding to promoter regions in genes known as hypoxia-response elements (HREs). HIF- $1 \alpha$ binds to the HRE located within the vascular endothelial growth factor (VEGF) promoter to increase expression of VEGF and drive angiogenesis. miR-100 derived from MSCs was shown to decrease expression and protein levels of mTOR, HIF-1 $\alpha$, and VEGF in MDA-MB-231 and MCF-7 breast cancer cell lines (known to overexpress VEGF) when co-cultured with MSCs containing miR-100 (146).

An opposing conclusion was made regarding the therapeutic use of MSC-derived exosomal ncRNA and cancer, whereby expression of various lncRNAs (AGAP2-AS1 and HCP5) promoted stemness and drug resistance in breast cancer and gastric cancer, respectively. Han et al. (140) found that AGAP2AS1 lncRNA was upregulated in trastuzumab-resistant breast cancer cells co-cultured with MSCs harboring lncRNA AGAP2AS1. AGAP2-AS1 was shown to interact directly with HuR (known to stabilize CPT1 mRNA) and increase fatty acid oxidation (FAO) to promote stemness and drug resistance. $\mathrm{Wu}$ et al. found that MSC-derived HCP5 lncRNA increased FAO in gastric cancer cell lines via sponging to miR-3619-5p (found to inhibit expression of CPT1) by affecting the AMPK pathway via PPARGC1A and PGC1 $\alpha$, leading to stemness and chemo-resistance. Overexpression of miR-3619-5p significantly decreased expression of PPARGC1A, which decreased PGC1 $\alpha$ (product of AMPK pathway), a transcriptional co-activator of FAO genes (137).

The regulation of cancer progression and inhibition via modulation of oncogenic pathways using MCS-derived ncRNA is an area that must be studied in greater depth. A solid understanding of ncRNA targets is needed to come to a consensus about the potential use, safety, and benefits of using exosomederived ncRNAs in cancer therapy.

\section{OTHER DISEASES}

In the retina, the exosomes derived from human U-MSCs (huc-MSCs) expressing miR-126 demonstrated remarkable therapeutic effects by decreasing hyperglycemia associated with retinal inflammation by downregulating the high-mobility group 
box 1 expression (HMGB1) in diabetic rats and human retinal endothelial cells (HREC) (147). In a murine subretinal fibrosis model, huc-MSCs exosomes containing miR-27b-3p could mitigate subretinal fibrosis by reversing the process of epithelial-mesenchymal transition (EMT) induced by TGF$\beta 2$ via inhibiting homeobox protein Hox-C6 (HOXC6) (148). The exosomal lncRNA H19 contributed to wound healing by preventing the apoptosis and inflammation of fibroblasts by impairing miR-152-3p-mediated PTEN inhibition in diabetes and foot ulcer disease (DFU) (149). A study of huc-MSCs in an experimental rat model of acute lung injury (ALI) revealed that exosomal miR-22-3p suppressed pathological changes, apoptosis, and NF- $\kappa \mathrm{B}$ expression in LPS-treated rats (150).

In summary, MSCs improve the regenerative potential in the above-mentioned diseases by transporting the exosomes enriched ncRNAs having anti-apoptotic, and anti-inflammatory effect.

\section{POTENTIAL CHALLENGES AND PROSPECTS ASSOCIATED WITH MSC-Exo-nCRNA}

The MSCs exosomes impart the regenerative effect on the target cells via ncRNAs, possibly making them a promising candidate in the tissue engineering field. However, the mechanisms associated with the ncRNAs packaging, uptake, release, and the underlying regulatory mechanisms are still in their infancy.

Many autoimmune diseases are characterized by an activated inflammatory state resulting in infiltration of the autoreactive immune cells damaging the tissues. Interestingly, MSCs-Exos in many autoimmune diseases have successfully induced immune polarization from an inflammatory to an anti-inflammatory state, mitigating the disease pathogenesis. Much of this might be mediated by the ncRNA repertoire in the exosomes. So, first, it's essential to determine the driving mechanism behind packaging the altered ncRNAs into the exosomes under a specific disease state. Secondly, it would be important to decipher the feedback mechanisms by which specific immune cells can control the exosome release as priming the MSCs to release the exosome more efficiently and enhance the therapeutic efficacy under disease conditions.

In addition to the challenges mentioned above, the low yield of exosomes/MSCs, their purification, storage, optimized dose to

\section{REFERENCES}

1. Fry DE, Pearlstein L, Fulton RL, Polk HC Jr. Multiple system organ failure. The role of uncontrolled infection. Arch Surg. (1980) 115:136-40. doi: 10.1001/archsurg.1980.013800200 06003

2. Ulvik A, Kvale R, Wentzel-Larsen T, Flaatten H. Multiple organ failure after trauma affects even long-term survival and functional status. Crit Care. (2007) 11:R95. doi: 10.1186/cc6111

3. Dewar DC, Mackay P, Balogh Z. Epidemiology of post-injury multiple organ failure in an Australian trauma system. ANZ J Surg. (2009) 79:4316. doi: 10.1111/j.1445-2197.2009.04968.x be administered would need further development. Therefore, the clinical application must scale up the production of MSCs-Exos without compromising their purity and stability. The concerns raised above impose significant challenges before bringing MSCsExos into clinical practice. Nevertheless, working on few of these domains might improve their regenerative potential.

\section{CONCLUSION}

The potential of the exosomes to recapitulate the therapeutic properties of the stem cells has paved the path toward cell-free alternative therapy, allowing researchers to use them in a multiple diseases and disorders. The underlying factors and the associated mechanisms contributing to the exosomes' regenerative effects have been an area of interest in the past few years. The recent studies depict that the regenerative effects of the exosomes can be ascribed to their ncRNA repertoire. Moreover, modulating the expression of the ncRNAs in the exosomes has allowed researchers to extensively evaluate the protective effects in tissue repair and regenerative medicine. It will be interesting to investigate how the modification in the intracellular and extracellular conditions of the MSCs itself would change the ncRNA repertoire. In addition to that, it would be important to investigate if these changes could eventually generate exosomes having a homogenous expression of the ncRNAs and could be used safely in the clinics.

\section{AUTHOR CONTRIBUTIONS}

$\mathrm{TP}$ and $\mathrm{AD}$ conceived the original idea. TP and MJ collected and prepared the literature, reviewed the literature, and wrote the original manuscript. $\mathrm{TP}, \mathrm{AD}, \mathrm{MJ}$, and $\mathrm{ZB}$ contributed to interpretation of the literature. All authors read, discussed, and revised the initial manuscript and read, approved, and contributed to the final manuscript.

\section{FUNDING}

This work was supported in part by DST-UKEIRI DST/INT/UK/P-50/2012 to $\mathrm{AD}$ and $\mathrm{TP}$ and by the grant P01GM066730 from the National Institutes of Health, Bethesda, $\mathrm{MD}$, to $\mathrm{ZB}$ and TP. The funding body had no role in the design of the study, collection, analysis, interpretation of data, and in writing the manuscript.
4. Abouna GM. Organ shortage crisis: problems and possible solutions. Transplant Proc. (2008) 40:34-8. doi: 10.1016/j.transproceed.2007.11.067

5. Sellares J, de Freitas DG, Mengel M, Reeve J, Einecke G, Sis B, et al. Understanding the causes of kidney transplant failure: the dominant role of antibody-mediated rejection and nonadherence. Am J Transplant. (2012) 12:388-99. doi: 10.1111/j.1600-6143.2011.03840.x

6. Becker LE, Morath C, Suesal C. Immune mechanisms of acute and chronic rejection. Clin Biochem. (2016) 49:3203. doi: 10.1016/j.clinbiochem.2016.02.001

7. Choudhary NS, Saigal S, Bansal RK, Saraf N, Gautam D, Soin AS. Acute and chronic rejection after liver transplantation: what a clinician needs to know. J Clin Exp Hepatol. (2017) 7:358-66. doi: 10.1016/j.jceh.2017.10.003 
8. Vlaovic PD, Devins GM, Abbey S, Wright E, Robinette MA. Psychosocial impact of renal donation. Can J Urol. (1999) 6:859-64.

9. Klarenbach S, Garg AX, Vlaicu S. Living organ donors face financial barriers: a national reimbursement policy is needed. CMAJ. (2006) 174:7978. doi: $10.1503 / \mathrm{cmaj} .051168$

10. Orlando G, Wood KJ, Stratta RJ, Yoo JJ, Atala A, Soker S. Regenerative medicine and organ transplantation: past, present, and future. Transplantation. (2011) 91:1310-7. doi: 10.1097/TP.0b013e318219ebb5

11. Edgar L, Pu T, Porter B, Aziz JM, La Pointe C, Asthana A, et al. Regenerative medicine, organ bioengineering and transplantation. Br J Surg. (2020) 107:793-800. doi: 10.1002/bjs.11686

12. Jain A, Bansal R. Applications of regenerative medicine in organ transplantation. J Pharm Bioallied Sci. (2015) 7:18894. doi: 10.4103/0975-7406.160013

13. Wilke MM, Nydam DV, Nixon AJ. Enhanced early chondrogenesis in articular defects following arthroscopic mesenchymal stem cell implantation in an equine model. J Orthop Res. (2007) 25:913-25. doi: 10.1002/jor.20382

14. Chong JJ, Yang X, Don CW, Minami E, Liu YW, Weyers JJ, et al. Human embryonic-stem-cell-derived cardiomyocytes regenerate nonhuman primate hearts. Nature. (2014) 510:273-7. doi: 10.1038/nature13233

15. Daadi MM, Grueter BA, Malenka RC, Redmond DE Jr, Steinberg GK. Dopaminergic neurons from midbrain-specified human embryonic stem cell-derived neural stem cells engrafted in a monkey model of Parkinson's disease. PLoS One. (2012) 7:e41120. doi: 10.1371/journal.pone.0041120

16. Zhou L, Wang W, Liu Y, Fernandez de Castro J, Ezashi T, Telugu BP, et al. Differentiation of induced pluripotent stem cells of swine into rod photoreceptors and their integration into the retina. Stem Cells. (2011) 29:972-80. doi: 10.1002/stem.637

17. Musial-Wysocka A, Kot M, Majka M. The pros and cons of mesenchymal stem cell-based therapies. Cell Transplant. (2019) 28:801-12. doi: 10.1177/0963689719837897

18. Karussis D, Karageorgiou C, Vaknin-Dembinsky A, Gowda-Kurkalli B, Gomori JM, Kassis I, et al. Safety and immunological effects of mesenchymal stem cell transplantation in patients with multiple sclerosis and amyotrophic lateral sclerosis. Arch Neurol. (2010) 67:118794. doi: 10.1001/archneurol.2010.248

19. Oh KW, Moon C, Kim HY, Oh SI, Park J, Lee JH, et al. Phase I trial of repeated intrathecal autologous bone marrow-derived mesenchymal stromal cells in amyotrophic lateral sclerosis. Stem Cells Transl Med. (2015) 4:5907. doi: 10.5966/sctm.2014-0212

20. Lalu MM, McIntyre L, Pugliese C, Fergusson D, Winston BW, Marshall JC, et al. Safety of cell therapy with mesenchymal stromal cells (SafeCell): a systematic review and meta-analysis of clinical trials. PLoS One. (2012) 7:e47559. doi: 10.1371/journal.pone.0047559

21. Pittenger MF, Discher DE, Peault BM, Phinney DG, Hare JM, Caplan AI. Mesenchymal stem cell perspective: cell biology to clinical progress. NPJ Regen Med. (2019) 4:22. doi: 10.1038/s41536-019-0083-6

22. Phinney DG, Pittenger MF. Concise review: MSC-derived exosomes for cell-free therapy. Stem Cells. (2017) 35:851-8. doi: 10.1002/stem.2575

23. Nakamura Y, Miyaki S, Ishitobi H, Matsuyama S, Nakasa T, Kamei N, et al. Mesenchymal-stem-cell-derived exosomes accelerate skeletal muscle regeneration. FEBS Lett. (2015) 589:1257-65. doi: 10.1016/j.febslet.2015.03.031

24. Furuta T, Miyaki S, Ishitobi H, Ogura T, Kato Y, Kamei N, et al. Mesenchymal stem cell-derived exosomes promote fracture healing in a mouse model. Stem Cells Transl Med. (2016) 5:1620-30. doi: 10.5966/sctm.20 15-0285

25. Reis M, Mavin E, Nicholson L, Green K, Dickinson AM, Wang XN. Mesenchymal stromal cell-derived extracellular vesicles attenuate dendritic cell maturation and function. Front Immunol. (2018) 9:2538. doi: 10.3389/fimmu.2018.02538

26. Kaur S, Abu-Shahba AG, Paananen RO, Hongisto H, Hiidenmaa $\mathrm{H}$, Skottman $\mathrm{H}$, et al. Small non-coding RNA landscape of extracellular vesicles from human stem cells. Sci Rep. (2018) 8:15503. doi: 10.1038/s41598-018-33899-6

27. Ferguson SW, Wang J, Lee CJ, Liu M, Neelamegham S, Canty JM, et al. The microRNA regulatory landscape of MSC-derived exosomes: a systems view. Sci Rep. (2018) 8:1419. doi: 10.1038/s41598-018-19581-x
28. Kishore R, Khan M. More than tiny sacks: stem cell exosomes as cell-free modality for cardiac repair. Circ Res. (2016) 118:33043. doi: 10.1161/CIRCRESAHA.115.307654

29. Ragni E, Colombini A, De Luca P, Libonati F, Vigano M, Perucca Orfei C, et al. miR-103a-3p and miR-22-5p are reliable reference genes in extracellular vesicles from cartilage, adipose tissue, and bone marrow cells. Front Bioeng Biotechnol. (2021) 9:632440. doi: 10.3389/fbioe.2021.632440

30. Wang A, Liu J, Zhuang X, Yu S, Zhu S, Liu Y, et al. Identification and comparison of piRNA expression profiles of exosomes derived from human stem cells from the apical papilla and bone marrow mesenchymal stem cells. Stem Cells Dev. (2020) 29:511-20. doi: 10.1089/scd.2019.0277

31. Petrenko Y, Vackova I, Kekulova K, Chudickova M, Koci Z, Turnovcova $\mathrm{K}$, et al. A comparative analysis of multipotent mesenchymal stromal cells derived from different sources, with a focus on neuroregenerative potential. Sci Rep. (2020) 10:4290. doi: 10.1038/s41598-020-61167-z

32. Segaloff DL, Limbird LE. The cAMP-Dependent induction of LH receptors in primary cultures of porcine granulosa cells is not due to the expression of an intracellular pool of LH receptors. Endocrinology. (1983) 113:8257. doi: 10.1210/endo-113-2-825

33. Gnecchi M, Melo LG. Bone marrow-derived mesenchymal stem cells: isolation, expansion, characterization, viral transduction, and production of conditioned medium. Methods Mol Biol. (2009) 482:281-94. doi: 10.1007/978-1-59745-060-7_18

34. Orbay H, Tobita M, Mizuno H. Mesenchymal stem cells isolated from adipose and other tissues: basic biological properties and clinical applications. Stem Cells Int. (2012) 2012:461718. doi: 10.1155/2012/461718

35. Miao Z, Jin J, Chen L, Zhu J, Huang W, Zhao J, et al. Isolation of mesenchymal stem cells from human placenta: comparison with human bone marrow mesenchymal stem cells. Cell Biol Int. (2006) 30:6817. doi: 10.1016/j.cellbi.2006.03.009

36. Tsai MS, Lee JL, Chang YJ, Hwang SM. Isolation of human multipotent mesenchymal stem cells from second-trimester amniotic fluid using a novel two-stage culture protocol. Hum Reprod. (2004) 19:14506. doi: 10.1093/humrep/deh279

37. Huang GT, Gronthos S, Shi S. Mesenchymal stem cells derived from dental tissues vs. those from other sources: their biology and role in regenerative medicine. J Dent Res. (2009) 88:792-806. doi: 10.1177/0022034509340867

38. Rastegar F, Shenaq D, Huang J, Zhang W, Zhang BQ, He BC, et al. Mesenchymal stem cells: molecular characteristics and clinical applications. World J Stem Cells. (2010) 2:67-80. doi: 10.4252/wjsc.v2.i4.67

39. Jones EA, Kinsey SE, English A, Jones RA, Straszynski L, Meredith DM, et al. Isolation and characterization of bone marrow multipotential mesenchymal progenitor cells. Arthritis Rheum. (2002) 46:3349-60. doi: 10.1002/art.10696

40. Buhring HJ, Battula VL, Treml S, Schewe B, Kanz L, Vogel W. Novel markers for the prospective isolation of human MSC. Ann N Y Acad Sci. (2007) 1106:262-71. doi: 10.1196/annals.1392.000

41. Birmingham E, Niebur GL, McHugh PE, Shaw G, Barry FP, McNamara LM. Osteogenic differentiation of mesenchymal stem cells is regulated by osteocyte and osteoblast cells in a simplified bone niche. Eur Cell Mater. (2012) 23:13-27. doi: 10.22203/eCM.v023a02

42. Solchaga LA, Penick KJ, Welter JF. Chondrogenic differentiation of bone marrow-derived mesenchymal stem cells: tips and tricks. Methods Mol Biol. (2011) 698:253-78. doi: 10.1007/978-1-60761-999-4_20

43. Scott MA, Nguyen VT, Levi B, James AW. Current methods of adipogenic differentiation of mesenchymal stem cells. Stem Cells Dev. (2011) 20:1793804. doi: $10.1089 /$ scd.2011.0040

44. Si Y, Zhao Y, Hao H, Liu J, Guo Y, Mu Y, et al. Infusion of mesenchymal stem cells ameliorates hyperglycemia in type 2 diabetic rats: identification of a novel role in improving insulin sensitivity. Diabetes. (2012) 61:161625. doi: $10.2337 / \mathrm{db} 11-1141$

45. Jurewicz M, Yang S, Augello A, Godwin JG, Moore RF, Azzi J, et al. Congenic mesenchymal stem cell therapy reverses hyperglycemia in experimental type 1 diabetes. Diabetes. (2010) 59:3139-47. doi: 10.2337/db10-0542

46. Hare JM, Fishman JE, Gerstenblith G, DiFede Velazquez DL, Zambrano JP, Suncion VY, et al. Comparison of allogeneic vs autologous bone marrowderived mesenchymal stem cells delivered by transendocardial injection in patients with ischemic cardiomyopathy: the POSEIDON randomized trial. JAMA. (2012) 308:2369-79. doi: 10.1001/jama.2012.25321 
47. Lee JW, Lee SH, Youn YJ, Ahn MS, Kim JY, Yoo BS, et al. A randomized, open-label, multicenter trial for the safety and efficacy of adult mesenchymal stem cells after acute myocardial infarction. J Korean Med Sci. (2014) 29:2331. doi: 10.3346/jkms.2014.29.1.23

48. van Buul GM, Villafuertes E, Bos PK, Waarsing JH, Kops N, Narcisi R, et al. Mesenchymal stem cells secrete factors that inhibit inflammatory processes in short-term osteoarthritic synovium and cartilage explant culture. Osteoarthritis Cartilage. (2012) 20:1186-96. doi: 10.1016/j.joca.2012.06.003

49. Lee JK, Jin HK, Bae JS. Bone marrow-derived mesenchymal stem cells reduce brain amyloid-beta deposition and accelerate the activation of microglia in an acutely induced Alzheimer's disease mouse model. Neurosci Lett. (2009) 450:136-41. doi: 10.1016/j.neulet.2008.11.059

50. Tsiapalis D, O’Driscoll L. Mesenchymal stem cell derived extracellular vesicles for tissue engineering and regenerative medicine applications. Cells. (2020) 9:991. doi: 10.3390/cells9040991

51. Shifrin DA Jr, Demory Beckler M, Coffey RJ, Tyska MJ. Extracellular vesicles: communication, coercion, and conditioning. Mol Biol Cell. (2013) 24:12539. doi: 10.1091/mbc.e12-08-0572

52. Zhang Y, Liu Y, Liu H, Tang WH. Exosomes: biogenesis, biologic function and clinical potential. Cell Biosci. (2019) 9:19. doi: 10.1186/s13578-019-0282-2

53. Mostafazadeh M, Samadi N, Kahroba H, Baradaran B, Haiaty S, Nouri M. Potential roles and prognostic significance of exosomes in cancer drug resistance. Cell Biosci. (2021) 11:1. doi: 10.1186/s13578-020-00515-y

54. Colombo M, Raposo G, Thery C. Biogenesis, secretion, and intercellular interactions of exosomes and other extracellular vesicles. Annu Rev Cell Dev Biol. (2014) 30:255-89. doi: 10.1146/annurev-cellbio-101512-122326

55. Joo HS, Suh JH, Lee HJ, Bang ES, Lee JM. Current knowledge and future perspectives on mesenchymal stem cell-derived exosomes as a new therapeutic agent. Int J Mol Sci. (2020) 21:727. doi: 10.3390/ijms21030727

56. Wang J, Bonacquisti EE, Brown AD, Nguyen J. Boosting the biogenesis and secretion of mesenchymal stem cell-derived exosomes. Cells. (2020) 9:660. doi: 10.3390/cells9030660

57. Alcayaga-Miranda F, Varas-Godoy M, Khoury M. Harnessing the angiogenic potential of stem cell-derived exosomes for vascular regeneration. Stem Cells Int. (2016) 2016:3409169. doi: 10.1155/2016/3409169

58. Lai RC, Arslan F, Lee MM, Sze NS, Choo A, Chen TS, et al. Exosome secreted by MSC reduces myocardial ischemia/reperfusion injury. Stem Cell Res. (2010) 4:214-22. doi: 10.1016/j.scr.2009.12.003

59. Huang P, Wang L, Li Q, Xu J, Xu J, Xiong Y, et al. Combinatorial treatment of acute myocardial infarction using stem cells and their derived exosomes resulted in improved heart performance. Stem Cell Res Ther. (2019) 10:300. doi: 10.1186/s13287-019-1353-3

60. Zhang Z, Yang J, Yan W, Li Y, Shen Z, Asahara T. Pretreatment of cardiac stem cells with exosomes derived from mesenchymal stem cells enhances myocardial repair. J Am Heart Assoc. (2016) 5:e002856. doi: 10.1161/JAHA.115.002856

61. Rong X, Liu J, Yao X, Jiang T, Wang Y, Xie F. Human bone marrow mesenchymal stem cells-derived exosomes alleviate liver fibrosis through the Wnt/beta-catenin pathway. Stem Cell Res Ther. (2019) 10:98. doi: 10.1186/s13287-019-1204-2

62. Jung JW, Kwon M, Choi JC, Shin JW, Park IW, Choi BW, et al. Familial occurrence of pulmonary embolism after intravenous, adipose tissue-derived stem cell therapy. Yonsei Med J. (2013) 54:1293-6. doi: 10.3349/ymj.2013.54.5.1293

63. Cosenza S, Toupet K, Maumus M, Luz-Crawford P, Blanc-Brude $\mathrm{O}$, Jorgensen $\mathrm{C}$, et al. Mesenchymal stem cells-derived exosomes are more immunosuppressive than microparticles in inflammatory arthritis. Theranostics. (2018) 8:1399-410. doi: 10.7150/thno. 21072

64. Burrello J, Monticone S, Gai C, Gomez Y, Kholia S, Camussi G. Stem cellderived extracellular vesicles and immune-modulation. Front Cell Dev Biol. (2016) 4:83. doi: 10.3389/fcell.2016.00083

65. Chen TS, Lai RC, Lee MM, Choo AB, Lee CN, Lim SK. Mesenchymal stem cell secretes microparticles enriched in pre-microRNAs. Nucleic Acids Res. (2010) 38:215-24. doi: 10.1093/nar/gkp857

66. Di Trapani M, Bassi G, Midolo M, Gatti A, Kamga PT, Cassaro A, et al. Differential and transferable modulatory effects of mesenchymal stromal cell-derived extracellular vesicles on T, B and NK cell functions. Sci Rep. (2016) 6:24120. doi: 10.1038/srep24120

67. Palazzo AF, Lee ES. Non-coding RNA: what is functional and what is junk? Front Genet. (2015) 6:2. doi: 10.3389/fgene.2015.00002

68. Richard Boland C. Non-coding RNA: it's not junk. Dig Dis Sci. (2017) 62:1107-9. doi: 10.1007/s10620-017-4506-1

69. Pennisi E. Genomics. ENCODE project writes eulogy for junk DNA. Science. (2012) 337:1159:61. doi: 10.1126/science.337.6099.1159

70. Cech TR, Steitz JA. The noncoding RNA revolution-trashing old rules to forge new ones. Cell. (2014) 157:77-94. doi: 10.1016/j.cell.2014.03.008

71. Kopp F, Mendell JT. Functional classification and experimental dissection of long noncoding RNAs. Cell. (2018) 172:393407. doi: 10.1016/j.cell.2018.01.011

72. Lee JT. Epigenetic regulation by long noncoding RNAs. Science. (2012) 338:1435-9. doi: 10.1126/science.1231776

73. Pant T, Dhanasekaran A, Bai X, Zhao M, Thorp EB, Forbess JM, et al. Genome-wide differential expression profiling of lncRNAs and mRNAs associated with early diabetic cardiomyopathy. Sci Rep. (2019) 9:15345. doi: 10.1038/s41598-019-51872-9

74. Pant T, Dhanasekaran A, Zhao M, Thorp EB, Forbess JM, Bosnjak ZJ, et al. Identification and analysis of circulating long non-coding RNAs with high significance in diabetic cardiomyopathy. Sci Rep. (2021) 11:2571. doi: 10.1038/s41598-021-82345-7

75. Pant T, Dhanasekaran A, Fang J, Bai X, Bosnjak ZJ, Liang M, et al. Current status and strategies of long noncoding RNA research for diabetic cardiomyopathy. BMC Cardiovasc Disord. (2018) 18:197. doi: 10.1186/s12872-018-0939-5

76. Pant T, DiStefano JK, Logan S, Bosnjak ZJ. Emerging role of long noncoding RNAs in perioperative neurocognitive disorders and anestheticinduced developmental neurotoxicity. Anesth Analg. (2020) 132:1614-25. doi: 10.1213/ANE.0000000000005317

77. Harries LW. Long non-coding RNAs and human disease. Biochem Soc Trans. (2012) 40:902-6. doi: 10.1042/BST20120020

78. Liao W, Ning $\mathrm{Y}, \mathrm{Xu} \mathrm{HJ}$, Zou WZ, Hu J, Liu XZ, et al. BMSC-derived exosomes carrying microRNA-122-5p promote proliferation of osteoblasts in osteonecrosis of the femoral head. Clin Sci (Lond). (2019) 133:195575. doi: 10.1042/CS20181064

79. Xu HJ, Liao W, Liu XZ, Hu J, Zou WZ, Ning Y, et al. Downregulation of exosomal microRNA-224-3p derived from bone marrow-derived mesenchymal stem cells potentiates angiogenesis in traumatic osteonecrosis of the femoral head. FASEB J. (2019) 33:8055-68. doi: 10.1096/fj.201801618RRR

80. Kuang MJ, Zhang KH, Qiu J, Wang AB, Che WW, Li XM, et al. Exosomal miR-365a-5p derived from HUC-MSCs regulates osteogenesis in GIONFH through the Hippo signaling pathway. Mol Ther Nucleic Acids. (2021) 23:565-76. doi: 10.1016/j.omtn.2020.12.006

81. Yang BC, Kuang MJ, Kang JY, Zhao J, Ma JX, Ma XL. Human umbilical cord mesenchymal stem cell-derived exosomes act via the miR-1263/Mob1/Hippo signaling pathway to prevent apoptosis in disuse osteoporosis. Biochem Biophys Res Commun. (2020) 524:883-9. doi: 10.1016/j.bbrc.2020.02.001

82. Li L, Zhou X, Zhang JT, Liu AF, Zhang C, Han JC, et al. Exosomal miR186 derived from BMSCs promote osteogenesis through hippo signaling pathway in postmenopausal osteoporosis. J Orthop Surg Res. (2021) 16:23. doi: 10.1186/s13018-020-02160-0

83. Xu T, Luo Y, Wang J, Zhang N, Gu C, Li L, et al. Exosomal miRNA128-3p from mesenchymal stem cells of aged rats regulates osteogenesis and bone fracture healing by targeting Smad5. J Nanobiotechnology. (2020) 18:47. doi: 10.1186/s12951-020-00601-w

84. Liu W, Li L, Rong Y, Qian D, Chen J, Zhou Z, et al. Hypoxic mesenchymal stem cell-derived exosomes promote bone fracture healing by the transfer of miR-126. Acta Biomater. (2020) 103:196-212. doi: 10.1016/j.actbio.2019.12.020

85. Liu Y, Lin L, Zou R, Wen C, Wang Z, Lin F. MSC-derived exosomes promote proliferation and inhibit apoptosis of chondrocytes via lncRNAKLF3-AS1/miR-206/GIT1 axis in osteoarthritis. Cell Cycle. (2018) 17:241122. doi: 10.1080/15384101.2018.1526603

86. Wu J, Kuang L, Chen C, Yang J, Zeng WN, Li T, et al. miR-100-5p-abundant exosomes derived from infrapatellar fat pad MSCs protect articular cartilage 
and ameliorate gait abnormalities via inhibition of mTOR in osteoarthritis. Biomaterials. (2019) 206:87-100. doi: 10.1016/j.biomaterials.2019.03.022

87. Tao SC, Yuan T, Zhang YL, Yin WJ, Guo SC, Zhang CQ. Exosomes derived from miR-140-5p-overexpressing human synovial mesenchymal stem cells enhance cartilage tissue regeneration and prevent osteoarthritis of the knee in a rat model. Theranostics. (2017) 7:180-95. doi: 10.7150/thno.17133

88. Yan L, Liu G, Wu X. Exosomes derived from umbilical cord mesenchymal stem cells in mechanical environment show improved osteochondral activity via upregulation of LncRNA H19. J Orthop Translat. (2021) 26:11120. doi: $10.1016 /$ j.jot.2020.03.005

89. Qiu M, Liu D, Fu Q. MiR-129-5p shuttled by human synovial mesenchymal stem cell-derived exosomes relieves IL-1 $\beta$ induced osteoarthritis via targeting HMGB1. Life Sci. (2021) 269:118987. doi: 10.1016/j.lfs.2020.118987

90. Jin Z, Ren J, Qi S. Human bone mesenchymal stem cells-derived exosomes overexpressing microRNA-26a-5p alleviate osteoarthritis via down-regulation of PTGS2. Int Immunopharmacol. (2020) 78:105946. doi: 10.1016/j.intimp.2019.105946

91. Li Y, Yang R, Guo B, Zhang H, Zhang H, Liu S, et al. Exosomal miR301 derived from mesenchymal stem cells protects myocardial infarction by inhibiting myocardial autophagy. Biochem Biophys Res Commun. (2019) 514:323-8. doi: 10.1016/j.bbrc.2019.04.138

92. Chen Q, Liu Y, Ding X, Li Q, Qiu F, Wang M, et al. Bone marrow mesenchymal stem cell-secreted exosomes carrying microRNA-125b protect against myocardial ischemia reperfusion injury via targeting SIRT7. Mol Cell Biochem. (2020) 465:103-14. doi: 10.1007/s11010-019-03671-z

93. Zhu LP, Tian T, Wang JY, He JN, Chen T, Pan M, et al. Hypoxia-elicited mesenchymal stem cell-derived exosomes facilitates cardiac repair through miR-125b-mediated prevention of cell death in myocardial infarction. Theranostics. (2018) 8:6163-77. doi: 10.7150/thno.28021

94. Liu Y, Duan C, Liu W, Chen X, Wang Y, Liu X, et al. Upregulation of let-7f-2-3p by long noncoding RNA NEAT1 inhibits XPO1-mediated HAX-1 nuclear export in both in vitro and in vivo rodent models of doxorubicin-induced cardiotoxicity. Arch Toxicol. (2019) 93:326176. doi: 10.1007/s00204-019-02586-4

95. Xin H, Wang F, Li Y, Lu QE, Cheung WL, Zhang Y, et al. Secondary release of exosomes from astrocytes contributes to the increase in neural plasticity and improvement of functional recovery after stroke in rats treated with exosomes harvested from microRNA 133b-overexpressing multipotent mesenchymal stromal cells. Cell Transplant. (2017) 26:24357. doi: $10.3727 / 096368916$ X693031

96. Cai G, Cai G, Zhou H, Zhuang Z, Liu K, Pei S, et al. Mesenchymal stem cellderived exosome miR-542-3p suppresses inflammation and prevents cerebral infarction. Stem Cell Res Ther. (2021) 12:2. doi: 10.1186/s13287-020-02030-w

97. Zhao Y, Gan Y, Xu G, Hua K, Liu D. Exosomes from MSCs overexpressing microRNA-223-3p attenuate cerebral ischemia through inhibiting microglial M1 polarization mediated inflammation. Life Sci. (2020) 260:118403. doi: 10.1016/j.lfs.2020.118403

98. Liu X, Li Q, Niu X, Hu B, Chen S, Song W, et al. Exosomes secreted from human-induced pluripotent stem cell-derived mesenchymal stem cells prevent osteonecrosis of the femoral head by promoting angiogenesis. Int $J$ Biol Sci. (2017) 13:232-44. doi: 10.7150/ijbs.16951

99. Kuang MJ, Huang Y, Zhao XG, Zhang R, Ma JX, Wang DC, et al. Exosomes derived from Wharton's jelly of human umbilical cord mesenchymal stem cells reduce osteocyte apoptosis in glucocorticoid-induced osteonecrosis of the femoral head in rats via the miR-21-PTEN-AKT signalling pathway. Int J Biol Sci. (2019) 15:1861-71. doi: 10.7150/ijbs.32262

100. Liang B, Liang JM, Ding JN, Xu J, Xu JG, Chai YM. Dimethyloxaloylglycinestimulated human bone marrow mesenchymal stem cell-derived exosomes enhance bone regeneration through angiogenesis by targeting the AKT/mTOR pathway. Stem Cell Res Ther. (2019) 10:335. doi: 10.1186/s13287-019-1410-y

101. Zhang Y, Hao Z, Wang P, Xia Y, Wu J, Xia D, et al. Exosomes from human umbilical cord mesenchymal stem cells enhance fracture healing through HIF-1 $\alpha$-mediated promotion of angiogenesis in a rat model of stabilized fracture. Cell Prolif. (2019) 52:e12570. doi: 10.1111/cpr. 12570

102. Vonk LA, van Dooremalen SFJ, Liv N, Klumperman J, Coffer PJ, Saris DBF, et al. Mesenchymal stromal/stem cell-derived extracellular vesicles promote human cartilage regeneration in vitro. Theranostics. (2018) 8:90620. doi: 10.7150/thno. 20746

103. Li S, Stockl S, Lukas C, Gotz J, Herrmann M, Federlin M, et al. hBMSCderived extracellular vesicles attenuate IL-1 $\beta$-induced catabolic effects on OA-chondrocytes by regulating pro-inflammatory signaling pathways. Front Bioeng Biotechnol. (2020) 8:603598. doi: 10.3389/fbioe.2020.603598

104. Liu Y, Zou R, Wang Z, Wen C, Zhang F, Lin F. Exosomal KLF3-AS1 from hMSCs promoted cartilage repair and chondrocyte proliferation in osteoarthritis. Biochem J. (2018) 475:3629-38. doi: 10.1042/BCJ20180675

105. Song Q, Yu Y, Zhang X, Zhu Y, Luo Y, Yu T, et al. Value of MRI and diffusion weighted imaging in diagnosing normal-sized pelvic lymph nodes metastases in patients with cervical cancer. Br J Radiol. (2020). doi: 10.1259/bjr.20200203. [Epub ahead of print].

106. Yan L, Liu G, Wu X. The umbilical cord mesenchymal stem cell-derived exosomal lncRNA H19 improves osteochondral activity through miR-29b3p/FoxO3 axis. Clin Transl Med. (2021) 11:e255. doi: 10.1002/ctm2.255

107. Jin Z, Ren J, Qi S. Exosomal miR-9-5p secreted by bone marrow-derived mesenchymal stem cells alleviates osteoarthritis by inhibiting syndecan-1. Cell Tissue Res. (2020) 381:99-114. doi: 10.1007/s00441-020-03193-x

108. Schuleri KH, Boyle AJ, Hare JM. Mesenchymal stem cells for cardiac regenerative therapy. Handb Exp Pharmacol. (2007) 180:195-218. doi: 10.1007/978-3-540-68976-8_9

109. Sutton MG, Sharpe N. Left ventricular remodeling after myocardial infarction: pathophysiology and therapy. Circulation. (2000) 101:29818. doi: 10.1161/01.CIR.101.25.2981

110. Foglia MJ, Poss KD. Building and re-building the heart by cardiomyocyte proliferation. Development. (2016) 143:729-40. doi: 10.1242/dev.132910

111. Kikuchi K, Poss KD. Cardiac regenerative capacity and mechanisms. Аnnu Rev Cell Dev Biol. (2012) 28:71941. doi: 10.1146/annurev-cellbio-101011-155739

112. Szaraz P, Gratch YS, Iqbal F, Librach CL. In vitro differentiation of human mesenchymal stem cells into functional cardiomyocyte-like cells. J Vis Exp. (2017) 126:55757. doi: 10.3791/55757

113. Davidson SM, Takov K, Yellon DM. Exosomes and cardiovascular protection. Cardiovasc Drugs Ther. (2017) 31:77-86. doi: 10.1007/s10557-016-6698-6

114. Barile L, Lionetti V, Cervio E, Matteucci M, Gherghiceanu M, Popescu LM, et al. Extracellular vesicles from human cardiac progenitor cells inhibit cardiomyocyte apoptosis and improve cardiac function after myocardial infarction. Cardiovasc Res. (2014) 103:530-41. doi: 10.1093/cvr/cvul67

115. Gray WD, French KM, Ghosh-Choudhary S, Maxwell JT, Brown ME, Platt $\mathrm{MO}$, et al. Identification of therapeutic covariant microRNA clusters in hypoxia-treated cardiac progenitor cell exosomes using systems biology. Circ Res. (2015) 116:255-63. doi: 10.1161/CIRCRESAHA.116.304360

116. Wang Y, Zhao R, Liu D, Deng W, Xu G, Liu W, et al. Exosomes derived from miR-214-Enriched bone marrow-derived mesenchymal stem cells regulate oxidative damage in cardiac stem cells by targeting CaMKII. Oxid Med Cell Longev. (2018) 2018:4971261. doi: 10.1155/2018/4971261

117. Mao Q, Liang XL, Zhang CL, Pang YH, Lu YX. LncRNA KLF3-AS1 in human mesenchymal stem cell-derived exosomes ameliorates pyroptosis of cardiomyocytes and myocardial infarction through miR-138-5p/Sirt1 axis. Stem Cell Res Ther. (2019) 10:393. doi: 10.1186/s13287-019-1522-4

118. Li Y, Zhou J, Zhang O, Wu X, Guan X, Xue Y, et al. Bone marrow mesenchymal stem cells-derived exosomal microRNA185 represses ventricular remolding of mice with myocardial infarction by inhibiting SOCS2. Int Immunopharmacol. (2020) 80:106156. doi: 10.1016/j.intimp.2019.106156

119. Bi S, Liu Z, Wu Z, Wang Z, Liu X, Wang S, et al. SIRT7 antagonizes human stem cell aging as a heterochromatin stabilizer. Protein Cell. (2020) 11:483-504. doi: 10.1007/s13238-020-00728-4

120. Vakhrusheva O, Smolka C, Gajawada P, Kostin S, Boettger T, Kubin T, et al. Sirt7 increases stress resistance of cardiomyocytes and prevents apoptosis and inflammatory cardiomyopathy in mice. Circ Res. (2008) 102:70310. doi: 10.1161/CIRCRESAHA.107.164558

121. Liu C, Su C. Design strategies and application progress of therapeutic exosomes. Theranostics. (2019) 9:1015-28. doi: 10.7150/thno.30853

122. Sart S, Ma T, Li Y. Preconditioning stem cells for in vivo delivery. Biores Open Access. (2014) 3:137-49. doi: 10.1089/biores.2014.0012 
123. Alcendor RR, Gao S, Zhai P, Zablocki D, Holle E, Yu X, et al. Sirt1 regulates aging and resistance to oxidative stress in the heart. Circ Res. (2007) 100:1512-21. doi: 10.1161/01.RES.0000267723.65696.4a

124. Chen H, Xia W, Hou M. Correction to: LncRNA-NEAT1 from the competing endogenous RNA network promotes cardioprotective efficacy of mesenchymal stem cell-derived exosomes induced by macrophage migration inhibitory factor via the miR-142-3p/FOXO1 signaling pathway. Stem Cell Res Ther. (2020) 11:376. doi: 10.1186/s13287-020-01898-y

125. Virani SS, Alonso A, Benjamin EJ, Bittencourt MS, Callaway CW, Carson AP, et al. Heart disease and stroke statistics-2020 update: a report from the American Heart Association. Circulation. (2020) 141:e139e596. doi: 10.1161/CIR.0000000000000746

126. Hui C, Tadi P, Patti L. Ischemic Stroke. Treasure Island, FL: StatPearls (2021).

127. Sayed MA, Eldahshan W, Abdelbary M, Pillai B, Althomali W, Johnson $\mathrm{MH}$, et al. Stroke promotes the development of brain atrophy and delayed cell death in hypertensive rats. Sci Rep. (2020) 10:20233. doi: 10.1038/s41598-020-75450-6

128. Xin H, Li Y, Liu Z, Wang X, Shang X, Cui Y, et al. MiR-133b promotes neural plasticity and functional recovery after treatment of stroke with multipotent mesenchymal stromal cells in rats via transfer of exosome-enriched extracellular particles. Stem Cells. (2013) 31:2737-46. doi: 10.1002/stem.1409

129. Li G, Ma Q, Wang R, Fan Z, Tao Z, Liu P, et al. Diagnostic and immunosuppressive potential of elevated mir-424 levels in circulating immune cells of ischemic stroke patients. Aging Dis. (2018) 9:17281. doi: 10.14336/AD.2017.0602

130. Xin H, Katakowski M, Wang F, Qian JY, Liu XS, Ali MM, et al. MicroRNA cluster miR-17-92 cluster in exosomes enhance neuroplasticity and functional recovery after stroke in rats. Stroke. (2017) 48:74753. doi: 10.1161/STROKEAHA.116.015204

131. Sessa F, Maglietta F, Bertozzi G, Salerno M, Di Mizio G, Messina G, et al. Human brain injury and miRNAs: an experimental study. Int J Mol Sci. (2019) 20:1546. doi: 10.3390/ijms20071546

132. Schilling M, Besselmann M, Leonhard C, Mueller M, Ringelstein EB, Kiefer R. Microglial activation precedes and predominates over macrophage infiltration in transient focal cerebral ischemia: a study in green fluorescent protein transgenic bone marrow chimeric mice. Exp Neurol. (2003) 183:2533. doi: 10.1016/S0014-4886(03)00082-7

133. Liu T, Zhang L, Joo D, Sun SC. NF-кB signaling in inflammation. Signal Transduct Target Ther. (2017) 2:17023. doi: 10.1038/sigtrans.2017.23

134. Bachiller S, Jimenez-Ferrer I, Paulus A, Yang Y, Swanberg M, Deierborg $\mathrm{T}$, et al. Microglia in neurological diseases: a road map to braindisease dependent-inflammatory response. Front Cell Neurosci. (2018) 12:488. doi: 10.3389/fncel.2018.00488

135. Xu Y, Liu N, Wei Y, Zhou D, Lin R, Wang X, et al. Anticancer effects of miR124 delivered by BM-MSC derived exosomes on cell proliferation, epithelial mesenchymal transition, and chemotherapy sensitivity of pancreatic cancer cells. Aging (Albany NY). (2020) 12:19660-76. doi: 10.18632/aging.103997

136. Xu H, Zhao G, Zhang Y, Jiang H, Wang W, Zhao D, et al. Mesenchymal stem cell-derived exosomal microRNA-133b suppresses glioma progression via Wnt/beta-catenin signaling pathway by targeting EZH2. Stem Cell Res Ther. (2019) 10:381. doi: 10.1186/s13287-019-1446-Z

137. Wu H, Liu B, Chen Z, Li G, Zhang Z. MSC-induced lncRNA HCP5 drove fatty acid oxidation through miR-3619-5p/AMPK/PGC1 $\alpha /$ CEBPB axis to promote stemness and chemo-resistance of gastric cancer. Cell Death Dis. (2020) 11:233. doi: 10.1038/s41419-020-2426-Z

138. Biswas S, Mandal G, Roy Chowdhury S, Purohit S, Payne KK, Anadon C, et al. Exosomes produced by mesenchymal stem cells drive differentiation of myeloid cells into immunosuppressive M2-polarized macrophages in breast cancer. J Immunol. (2019) 203:3447-60. doi: 10.4049/jimmunol.1900692

139. Sun Y, Wang P, Yang W, Shan Y, Zhang Q, Wu H. The role of IncRNA MSCAS1/miR-29b-3p axis-mediated CDK14 modulation in pancreatic cancer proliferation and Gemcitabine-induced apoptosis. Cancer Biol Ther. (2019) 20:729-39. doi: 10.1080/15384047.2018.1529121

140. Han J, Qu H, Han M, Ding Y, Xie M, Hu J, et al. MSC-induced lncRNA AGAP2-AS1 promotes stemness and trastuzumab resistance through regulating $\mathrm{CPT} 1$ expression and fatty acid oxidation in breast cancer. Oncogene. (2021) 40:833-47. doi: 10.1038/s41388-020-01574-8

141. Uras IZ, Moll HP, Casanova E. Targeting KRAS mutant non-smallcell lung cancer: past, present and future. Int J Mol Sci. (2020) 21:4325. doi: $10.3390 / \mathrm{ijms} 21124325$

142. Ghimessy A, Radeczky P, Laszlo V, Hegedus B, Renyi-Vamos F, Fillinger J, et al. Current therapy of KRAS-mutant lung cancer. Cancer Metastasis Rev. (2020) 39:1159-77. doi: 10.1007/s10555-020-09903-9

143. Huang X, Yan J, Zhang M, Wang Y, Chen Y, Fu X, et al. Targeting epigenetic crosstalk as a therapeutic strategy for EZH2-aberrant solid tumors. Cell. (2018) 175:186-99.e19. doi: 10.1016/j.cell.2018.08.058

144. Chang L, Lei X, Qin YU, Zhang X, Jin H, Wang C, et al. MicroRNA-133b inhibits cell migration and invasion by targeting matrix metalloproteinase 14 in glioblastoma. Oncol Lett. (2015) 10:2781-6. doi: 10.3892/ol.2015.3657

145. Li C, Liu Z, Yang K, Chen X, Zeng Y, Liu J, et al. miR-133b inhibits glioma cell proliferation and invasion by targeting Sirt1. Oncotarget. (2016) 7:36247-54. doi: 10.18632/oncotarget.9198

146. Pakravan K, Babashah S, Sadeghizadeh M, Mowla SJ, MossahebiMohammadi M, Ataei F, et al. MicroRNA-100 shuttled by mesenchymal stem cell-derived exosomes suppresses in vitro angiogenesis through modulating the mTOR/HIF-1 $\alpha /$ VEGF signaling axis in breast cancer cells. Cell Oncol (Dordr). (2017) 40:457-70. doi: 10.1007/s13402-017-0335-7

147. Zhang W, Wang Y, Kong Y. Exosomes derived from mesenchymal stem cells modulate miR-126 to ameliorate hyperglycemia-induced retinal inflammation via targeting HMGB1. Invest Ophthalmol Vis Sci. (2019) 60:294-303. doi: 10.1167/iovs.18-25617

148. Li D, Zhang J, Liu Z, Gong Y, Zheng Z. Human umbilical cord mesenchymal stem cell-derived exosomal miR-27b attenuates subretinal fibrosis via suppressing epithelial-mesenchymal transition by targeting HOXC6. Stem Cell Res Ther. (2021) 12:24. doi: 10.1186/s13287-020-02064-0

149. Li B, Luan S, Chen J, Zhou Y, Wang T, Li Z, et al. The MSC-derived exosomal lncRNA H19 promotes wound healing in diabetic foot ulcers by upregulating PTEN via microRNA-152-3p. Mol Ther Nucleic Acids. (2020) 19:814-26. doi: 10.1016/j.omtn.2019.11.034

150. Zheng Y, Liu J, Chen P, Lin L, Luo Y, Ma X, et al. Exosomal miR-22$3 \mathrm{p}$ from human umbilical cord blood-derived mesenchymal stem cells protects against lipopolysaccharid-induced acute lung injury. Life Sci. (2021) 269:119004. doi: 10.1016/j.lfs.2020.119004

Conflict of Interest: The authors declare that the research was conducted in the absence of any commercial or financial relationships that could be construed as a potential conflict of interest.

Publisher's Note: All claims expressed in this article are solely those of the authors and do not necessarily represent those of their affiliated organizations, or those of the publisher, the editors and the reviewers. Any product that may be evaluated in this article, or claim that may be made by its manufacturer, is not guaranteed or endorsed by the publisher.

Copyright (ㄷ) 2021 Pant, Juric, Bosnjak and Dhanasekaran. This is an open-access article distributed under the terms of the Creative Commons Attribution License (CC $B Y)$. The use, distribution or reproduction in other forums is permitted, provided the original author(s) and the copyright owner(s) are credited and that the original publication in this journal is cited, in accordance with accepted academic practice. No use, distribution or reproduction is permitted which does not comply with these terms. 